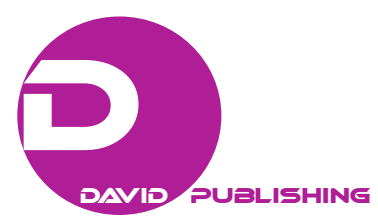

\title{
Analysis of Restricted Queueing Networks - A Blocking Approach
}

\author{
M. S. Sreekala and M. Manoharan \\ Department of Statistics \\ University of Calicut \\ Kerala - 673635 (India)
}

\begin{abstract}
In this paper we consider an open restricted queueing network with Poisson arrivals and exponential service times at stations and present an approximation method for analysis, made by node-by-node decomposition employing the concept of effective arrival rate and effective service rate. The steady state characteristics of the network system and of individual stations are obtained. Congestion due to blocking has been a severe problem in several health care studies, but among studies that apply queueing theory, those analyzing the blocking mechanism are still limited. This study applies to a health care system of infertility clinic considered as an open queueing network with blocking caused due to the capacity of the waiting space between stations. The method is illustrated with the help of an example network to study the efficiency of the approximation method. Finally a simulation study is performed to assess the transient behaviour of the network system. The blocking approach presented here for a simple model of a healthcare system can be easily adapted to any general open restricted queueing network.
\end{abstract}

Key words: queueing network, blocking, steady state, performance measures, finite capacity systems.

\section{Introduction}

Queueing networks are generally classified as restricted queueing networks or unrestricted queueing networks. Unrestricted queueing networks are those networks where all the queue nodes have unlimited capacity, while restricted queueing networks have finite capacity at some or all the queue nodes. Depending on the flow in the system, queueing networks can be further categorized as open unrestricted, closed unrestricted, open restricted and closed restricted.

The capacity restriction of a queueing network is a crucial aspect in its study and indeed there are many physical systems having finite capacity. In manufacturing systems, computer systems, communication networks, healthcare systems, supermarkets, airline terminals, and almost all physical environment, there are limitations on the capacity of the service provided as well as the accommodations of the customers. Majority of the research on queueing networks has concentrated on unrestricted networks due to their computational tractability and well behaved mathematical characteristics. In restricted queueing networks, "blocking" may occur due to restrictions on each queue length. The flow of customers from a node will be blocked if the corresponding destination node is full. Blocking in such a networks will be handled on the blocking mechanism that is being adopted. A blocking mechanism is a set of rules that determines the blocking and 
unblocking of a node. Blocking occurs when waiting spaces between stations are finite. Hunt [7] examined a finite buffer between two stations which is an exact solution method and computations are more complex. Hillier and Boling [6], Perros and Altiok [13], Takahashi et al. [14] considered similar situations and suggested different approximation methods. Korpororal et al. [11] analyzed the congestion level in penitetiary in Netherlands. Koizumi et al. [10] uses decomposition algorithm in mental health. Health care publications involving analysis of blocking are rare. El-Darzi et al. [3] and Cohen et al. [2] have used simulation studies and Hershey et al. [5] and Weiss and McClain [15] used mathematical studies for the analysis of queues with blocking. In a recent work of Manitz [12] multi-stage assembly-disassembly queueing networks with blocking after service have been analyzed using a decomposition approach to evaluate the performance of the network.

In this paper we present an analysis of restricted queueing network system with special reference to an example situation of a healthcare system. For such a system we analyze the congestion in the system by applying a queueing network model with blocking. Two performance indicators, the number of patients waiting to enter each type of facility in the system and the associated waiting time are derived in the steady state analysis. The additional feature of the network considered in this paper is in that all the feedback customers are combined and directed to the first station. This kind of feedback is prevalent and more realistic in infertility clinics and other health care systems. This study helps to reduce the unnecessary waiting time by changing the capacity as well as identifying the strategies to reducing queue length.

\subsection{Model description}

We consider a health care system of infertility clinic as an open queueing network system, where patients arrival at the clinic is according to a homogeneous Poisson process and get self-service with a general service time distribution for completing an initial non-clinical registration formality. According to the revised glossary of the International Committee for Monitoring Assisted Reproductive Technology (ICMART) and the World Health Organization (WHO) the clinical definition of infertility is "a disease of the reproductive system defined by the failure to achieve a clinical pregnancy after twelve months or more of regular unprotected sexual intercourse" (see Zegers-Hochschild et.al [16]). For the treatment of this disease the male and female partners are evaluated for infertility or subfertility using a variety of clinical interventions and also from certain laboratory evaluations on the individuals. At the initial registration facility, specific enquiries about lifestyle and sexual history of couples are collected to identify people who are less likely to conceive. After completing this formality they enter into the system, that is the external arrival node namely admission node $\left(S_{1}\right)$ which follows an $M|M| 1|\infty| F C F S$ schedule. Here the forms are checked by the server and the people are divided into two categories according to 'years after marriage' and readmission. Clinic uses this as the initial predictor and does not check the other details in the first node. 'Less than one year after marriage' $(<1)$ couples are routed to the second node namely counseling $\left(S_{2}\right)$ with probability $\alpha_{1}$. 'More than one year after marriage' couples are routed to the third node namely treatment $\left(S_{3}\right)$ for treatment with probability $\left(1-\alpha_{1}\right)$. In the former case, the details of the couples are checked and they undergo a counseling process. This node follows an $M|M| 1|K| F C F S$ schedule. After the counseling session, the couples who realize that they don't require treatment leave the system with probability $\alpha_{2}$. If they want treatment, directed to the third node with probability $\left(1-\alpha_{2}\right)$. The third node also follows an $M|M| 1|K| F C F S$ schedule. At this node couples are treated and they leave this system with probability $\alpha_{3}$. This type of leaving may be due to completion of 
treatment (such as one day treatment) or giving up of treatment. Some couples want more cycles of treatments and some may be mentally stressed after consultationand want counseling, so all such feedback couples are routed to fourth node namely readmission $\left(S_{4}\right)$ with probability $\left(1-\alpha_{3}\right)$. This node also follows $M|M| 1|K| F C F S$ schedule. The readmitted patients are admitted to their respective nodes through the admission node. So the couples readmitted for counseling are routed to the second node with probability $\alpha_{1}$ and the couples readmitted for further treatments are routed to the third node with probability $\left(1-\alpha_{1}\right)$ and the process continues.

\section{Methodological framework}

We consider an open queueing network consisting of four single server nodes/stations with finite and infinite capacities and the stations are designated by $S_{i}(i=1,2,3,4)$. Customers arrive to the system according to a homogeneous Poisson process with rate $\lambda$ and get self service for nonclinical registration with a general service time distribution. The customers are then entered to the first node $\left(S_{1}\right)$ with rate $\lambda$. We assume that the service times are exponentially distributed with rate $\mu_{1}, \mu_{2}, \mu_{3}$ and $\mu_{4}$ at the respective nodes $i=1,2,3,4$ and there is an infinite waiting space capacity at the first node and other three nodes are of finite capacities. A diagrammatic representation of the above healthcare queueing network is shown in Figure 1.

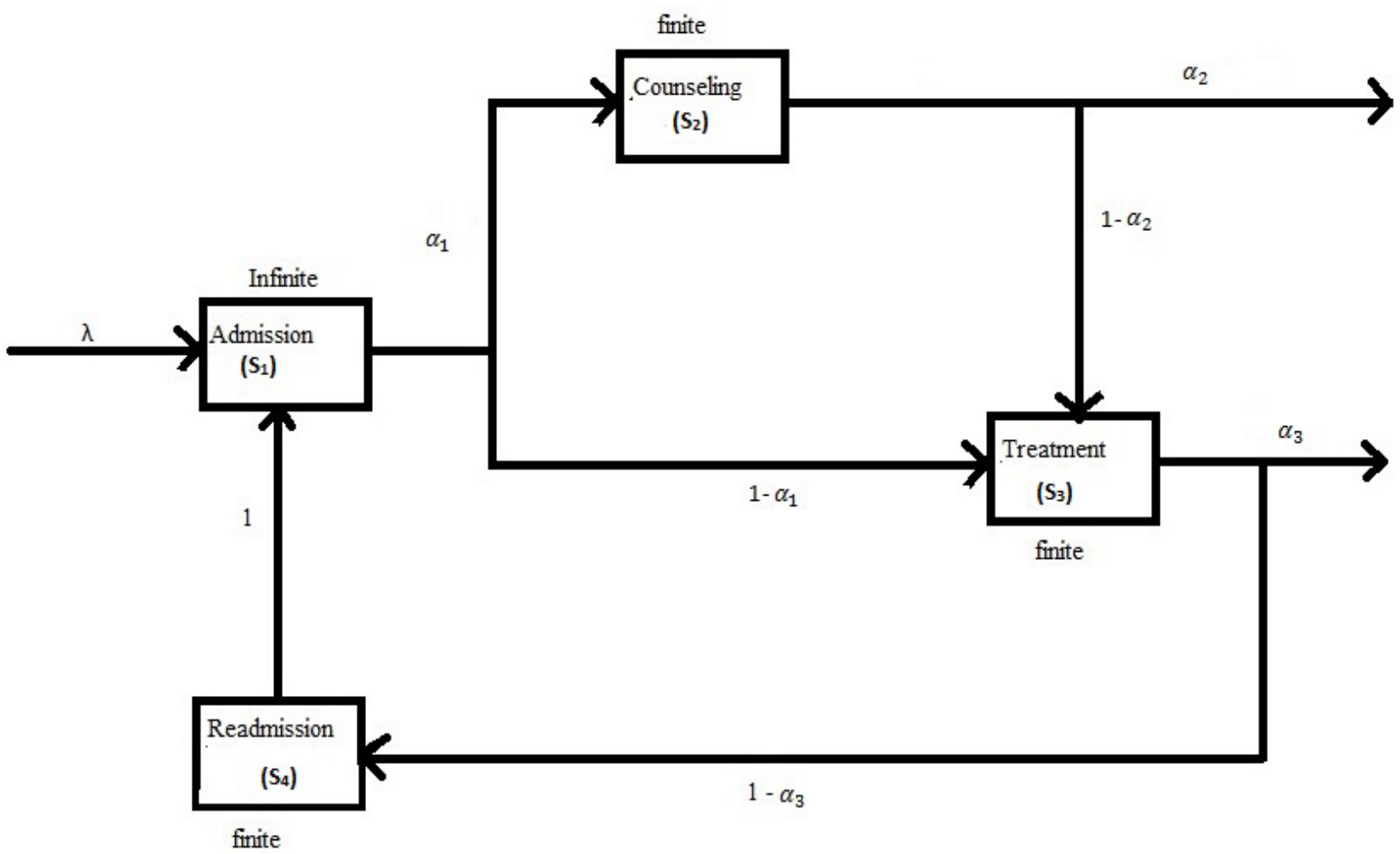

Figure 1. Health care queueing network with blocking.

Blocking occurs in this network because the waiting space between nodes two $\left(S_{2}\right)$ and three $\left(S_{3}\right)$ and three $\left(S_{3}\right)$ and four $\left(S_{4}\right)$ are finite. In this context it may noted that the arrival to each node is according to Poisson process with a certain rate. When blocking exists, congestion at any particular station could affect the 
congestion levels at all upstream stations. So using the concept of effective service time ( see Takahashi et al. [14]), service time is modified in order to corporate blocking between stations. Another assumption is that all patients arriving at any station in the system are treated equally without offering any priority. In the model, the stations $S_{2}, S_{3}$ and $S_{4}$ have finite capacity. So blocking occurs at the flows $S_{2} \rightarrow S_{3}$ and $S_{3} \rightarrow$ $S_{4}$. Blocking will not occur if the immediate station has infinite capacity. In our model station $S_{1}$ has infinite capacity. So the flow $S_{4} \rightarrow S_{1}$ is blocking free. Then the remaining flows $S_{1} \rightarrow S_{2}, S_{2} \rightarrow S_{3}$, $S_{3} \rightarrow S_{4}$ are modeled. When patients at $S_{1}$ find $S_{2}$ or $S_{3}$ full, they wait at station $S_{1}$. The Table 1 summarizes the types of congestion associated in this model.

Table 1

Types of congestions associated with each flow

\begin{tabular}{llll}
\hline Flow & Cause of congestion & Facing station & Congestion type \\
\hline$S_{1}$ to $S_{2}$ & $S_{2}$ is full & $S_{1}$ & Classic Congestion \\
$S_{1}$ to $S_{3}$ & $S_{3}$ is full & $S_{1}$ & Classic Congestion \\
$S_{2}$ to $S_{3}$ & $S_{3}$ is full & $S_{2}$ & Blocking \\
$S_{3}$ to $S_{4}$ & $S_{4}$ is full & $S_{3}$ & Blocking \\
$S_{4}$ to $S_{1}$ & Not applicable & Not applicable & No Congestion \\
\hline
\end{tabular}

\section{Steady-state Analysis}

Assuming that the inflows are compatible with system capacities that ensures the long run steady state behaviour of the model we first look into the analysis of the present network queueing model without considering blocking between stations. We shall then propose the modification in order to incorporate blocking. Following the steady state methodology for steady state analysis of queueing models (see Gross and Harris [4] and Bose [1] ) we proceed as follows.

\subsection{Steady-state analysis without blocking}

In our model, the routing probability matrix is expressed as follows:

$$
R=\left(r_{i j}\right)_{i, j=0,1,2,3,4}
$$

where $r_{i j}$ is the routing probability from station $i$ to station $j$. Here the station ' 0 ' represents outside system. Thus $r_{i j}$ is the fraction of patients departing from station $i$ to station $j$ in any given unit of time ( $i$, $j=0,1,2,3,4)$. Clearly the routing probability matrix corresponding to the network is :

$$
R=\left(\begin{array}{ccccc}
0 & 1 & 0 & 0 & 0 \\
0 & 0 & \alpha_{1} & 1-\alpha_{1} & 0 \\
\alpha_{2} & 0 & 0 & 1-\alpha_{2} & 0 \\
\alpha_{3} & 0 & 0 & 0 & 1-\alpha_{3} \\
0 & 1 & 0 & 0 & 0
\end{array}\right) .
$$

Based on the routing probabilities, the external arrival, the internal and the total arrival rates of the network system it may be noted that traffic equations (see Gross and Harris [4] and Bose [1]) for our system are: 


$$
\begin{gathered}
\lambda_{1}=\lambda+r_{41} \lambda_{4} \\
\lambda_{2}=r_{12} \lambda_{1} \\
\lambda_{3}=r_{13} \lambda_{1}+r_{23} \lambda_{2} \\
\lambda_{4}=r_{34} \lambda_{3} .
\end{gathered}
$$

Substituting the routing probabilities the above equations becomes

$$
\begin{gathered}
\lambda_{1}=\lambda+\lambda_{4} \\
\lambda_{2}=\alpha_{1} \lambda_{1} \\
\lambda_{3}=\left(1-\alpha_{1}\right) \lambda_{1}+\left(1-\alpha_{2}\right) \lambda_{2} \\
\lambda_{4}=\left(1-\alpha_{3}\right) \lambda_{2} .
\end{gathered}
$$

Solving the above set of equations we get,

$$
\begin{aligned}
& \lambda_{1}=\frac{\lambda}{\alpha_{3}\left(1-\alpha_{1} \alpha_{2}\right)+\alpha_{1} \alpha_{2}} \\
& \lambda_{2}=\frac{\alpha_{1} \lambda}{\alpha_{3}\left(1-\alpha_{1} \alpha_{2}\right)+\alpha_{1} \alpha_{2}} \\
& \lambda_{3}=\frac{\lambda\left(1-\alpha_{1} \alpha_{2}\right)}{\alpha_{3}\left(1-\alpha_{1} \alpha_{2}\right)+\alpha_{1} \alpha_{2}} \\
& \lambda_{4}=\frac{\lambda\left(1-\alpha_{3}\right)\left(1-\alpha_{1} \alpha_{2}\right)}{\alpha_{3}\left(1-\alpha_{1} \alpha_{2}\right)+\alpha_{1} \alpha_{2}} .
\end{aligned}
$$

We know that (Jackson [8], [9]) if there is an infinite buffer between stations in the network, each station can be solved independently. So we assume that there is an infinite buffer between stations. So each station can be solved independently by applying $M|M| 1 \mid \infty$ model. In a traditional queueing network model like an open Jackson network model without blocking, patients at a station arrive, receive services, and depart to the next station. In this system, the steady state of each station is analyzed without considering the impact of congestion at any particular station on the flows at other stations in the system.

\subsection{Average queue length and Average queue delay}

Expected queue length at station $i$ is obtained by the following formula 


$$
L_{i}^{q}=\frac{\rho_{i}{ }^{2}}{\left(1-\rho_{i}\right)},
$$

where $\rho_{i}=\frac{\lambda_{i}}{\mu_{i}}<1 \quad(i=1,2,3,4)$.

The expected steady-state waiting time to enter station $i$ can be obtained using Little's formula,

$$
W_{i}^{q}=\frac{L_{i}^{q}}{\lambda_{i}} .
$$

Therefore

$$
W_{i}^{q}=\frac{\rho_{i}{ }^{2}}{\lambda_{i}\left(1-\rho_{i}\right)}, \quad i=1,2,3,4 .
$$

\subsection{Steady-state analysis with blocking}

When stations are of finite capacity, congestion at any particular station could potentially affect congestion levels at all upstream stations. In this model we modify the Jackson approach to capture such interactions between stations. Using the concept of effective service time Takahashi et al. [14] we analyze this queueing network with blocking. Accordingly the effective service time is a combination of treatment time and blocked time. Treatment time represents the time between physical arrival to station $i$ and conceptual arrival to station $i+1$. Blocked time represents the time duration between physical exit from station $i$ and conceptual arrival to station $i+1$ and that is thus equivalent to the waiting time to enter station $i+1$ from station $i$. In networks without blocking, treatment time is equal to service times. For mathematical convenience, in our model we assume that the effective service time follows exponential distribution and mean effective service time at station $i$ is denoted as $\frac{1}{\tilde{\mu}_{i}}$. It is the mean treatment time $\left(\frac{1}{\mu_{i}}\right)$ plus the mean waiting time to enter station $i+1$ ( say $W_{i+1}$ ). In general effective service time is the convex combination of effective waiting times as givenby

$$
\frac{1}{\tilde{\mu}_{i}}=r_{i 0}\left(\frac{1}{\mu_{i}}\right)+\Sigma_{j} r_{i j}\left(\frac{1}{\mu_{i}}+W_{j}\right),
$$

where $r_{i 0}$ represents the routing probability of patients leaving the system from $i^{\text {th }}$ station without facing any wait and $r_{i j}$ represents the routing probability from $i^{\text {th }}$ station to the $j^{\text {th }}$ station. Hence in our system, stations $S_{2}$ and $S_{3}$ face blocking $(i=2,3)$.

Then, the effective service time corresponding to $S_{2}$ and $S_{3}$ are respectively,

$$
\begin{aligned}
& \frac{1}{\tilde{\mu}_{2}}=r_{20}\left(\frac{1}{\mu_{2}}\right)+r_{23}\left(\frac{1}{\mu_{2}}+W_{3}^{q}\right), \\
& \frac{1}{\tilde{\mu}_{3}}=r_{30}\left(\frac{1}{\mu_{3}}\right)+r_{34}\left(\frac{1}{\mu_{3}}+W_{4}^{q}\right) .
\end{aligned}
$$


By substituting the routing probabilities, the above equations become,

$$
\begin{aligned}
& \frac{1}{\tilde{\mu}_{2}}=\alpha_{2}\left(\frac{1}{\mu_{2}}\right)+\left(1-\alpha_{2}\right)\left(\frac{1}{\mu_{2}}+W_{3}^{q}\right), \\
& \frac{1}{\tilde{\mu}_{3}}=\alpha_{3}\left(\frac{1}{\mu_{3}}\right)+\left(1-\alpha_{3}\right)\left(\frac{1}{\mu_{3}}+W_{4}^{q}\right) .
\end{aligned}
$$

Using the last equations with (3.13) and (3.14), we get the steady state mean queue lengths and waiting times in terms of effective service times at the respective stations.

\section{Analysis of Stations}

We analyze the steady-state of each station using the node-by-node decomposition approximation technique proposed by Takahashi et al. [14]. In this approximation technique, the network is broken down into single nodes with revised service and arrival parameters which are then studied separately. So using the single node decomposition method this network is solved independently and individually from last station to first station. The steady state analysis of each finite station is done using single node decomposition approximation method with each station follows $M|M| 1 \mid \infty$ queue.

\subsection{Analysis of station four $\left(S_{4}\right)$}

In our system, station $S_{4}$ represents the patients those who want to re enter or readmit the clinic. Those patients from $S_{3}$ require reappearance at the node (feedback) will be directed to $S_{4}$. This is a finite capacity node, but its downstream node is $S_{1}$ which has infinite waiting space. So the patients at station $S_{4}$ do not face blocking. Thus, the effective service time corresponding to station $S_{4}$ is,

$$
\frac{1}{\tilde{\mu}_{4}}=\frac{1}{\mu_{4}} .
$$

So queue length and queue delay are obtained by using the formulas given in (3.13) and (3.14). The station $S_{4}$ receives patients only from station $S_{3}$. Therefore queue length at the station $S_{4}$, which is the steady-state number of blocked patients at $S_{3}$ waiting to enter $S_{4}$ denoted by $L_{34}^{q}$ is equal to $L_{4}^{q}$ itself.

\subsection{Analysis of station three $\left(S_{3}\right)$}

In this network, station $S_{3}$ represents station for treatment. There are two ways to enter station $S_{3}$, one directly from admission station $S_{1}$ and the other from counseling station $S_{2}$. When patients arriving from station $S_{2}$ experience blocking if the station $S_{3}$ is full. So the queue length and queue delay corresponding to station $S_{3}$ are obtained by solving (3.13) and (3.14) in terms of the effective service time expressed in (3.18).

The queue length at each station to enter station $S_{3}$ is obtained by the following equations.

$$
L_{13}^{q}=r_{13} L_{3}^{q},
$$

and

$$
L_{23}^{q}=r_{23} L_{3}^{q}
$$


Note that $L_{13}^{q}$ is the number of blocked patients at station $S_{1}$ waiting to enter station $S_{3}$ and $L_{23}^{q}$ is the number of blocked patients at station $S_{2}$ waiting to enter station $S_{3}$.

\subsection{Analysis of station two $\left(S_{2}\right)$}

In this network station $S_{2}$ is the station for counseling. Station $S_{2}$ receives patients only from admission station $S_{1}$, which includes new arrivals and readmitted patients. Thus queue length (3.13) and queue delay (3.14) of $S_{2}$ in termsof effective service time are calculated using (3.17).

The station $S_{2}$ receives patients only from station $S_{1}$. So the number of patients at $S_{1}$ waiting to enter $S_{2}$ is queue length of $S_{2}$ itself.

$$
L_{12}^{q}=L_{2}^{q}
$$

\section{Numerical Analysis}

A Matlab program has been developed for finding the steady state solution and the values of performance indicators (PI). The steady state probabilities and the performance measures with and without blocking are computed for the parameter values

$$
\lambda=0.4, \alpha_{1}=0.5, \alpha_{2}=0.3, \alpha_{3}=0.6, \frac{1}{\mu_{1}}=0.7, \frac{1}{\mu_{2}}=0.4, \frac{1}{\mu_{3}}=0.7, \frac{1}{\mu_{4}}=0.4 . \text { and the }
$$
summary of the analysis is given in Table 2 .

Table 2

Summary of steady state analysis

\begin{tabular}{llll}
\hline Station & PI & With Blocking & Without blocking \\
\hline$S_{2}$ & $L_{2}^{q}$ & 0.0555 & 0.0167 \\
& $W_{2}^{q}$ & 0.0831 & 0.0552 \\
$S_{3}$ & $L_{3}^{q}$ & 0.2143 & 0.2034 \\
& $W_{3}^{q}$ & 0.4160 & 0.3948 \\
& $L_{23}^{q}$ & 0.0630 & 0.0598 \\
& $L_{13}^{q}$ & 0.1261 & 0.1196 \\
$S_{4}$ & $L_{4}^{q}$ & 0.0074 & 0.0074 \\
& $W_{4}^{q}$ & 0.0359 & 0.0359 \\
& $L_{34}^{q}$ & 0.0074 & 0.0074 \\
\hline
\end{tabular}

Results show that the intensity of congestion is more at station $S_{3}$ than at station $S_{2}$. Waiting time to enter this station is high because at station $S_{3}$ there is a downstream of two stations $S_{1}$ and $S_{2}$. This situation may be due to the fact that the number of leaving patients is less than the number of readmitted patients. So by increasing the service rate of station $S_{3}$, waiting time of station $S_{3}$ is reduced and the system will be stabilized and bounded in this network. In figure 2 we plot the waiting time against the mean service time at station $S_{3}$. We observe that when service rate (reciprocal of mean service time) increases waiting time decreases. 


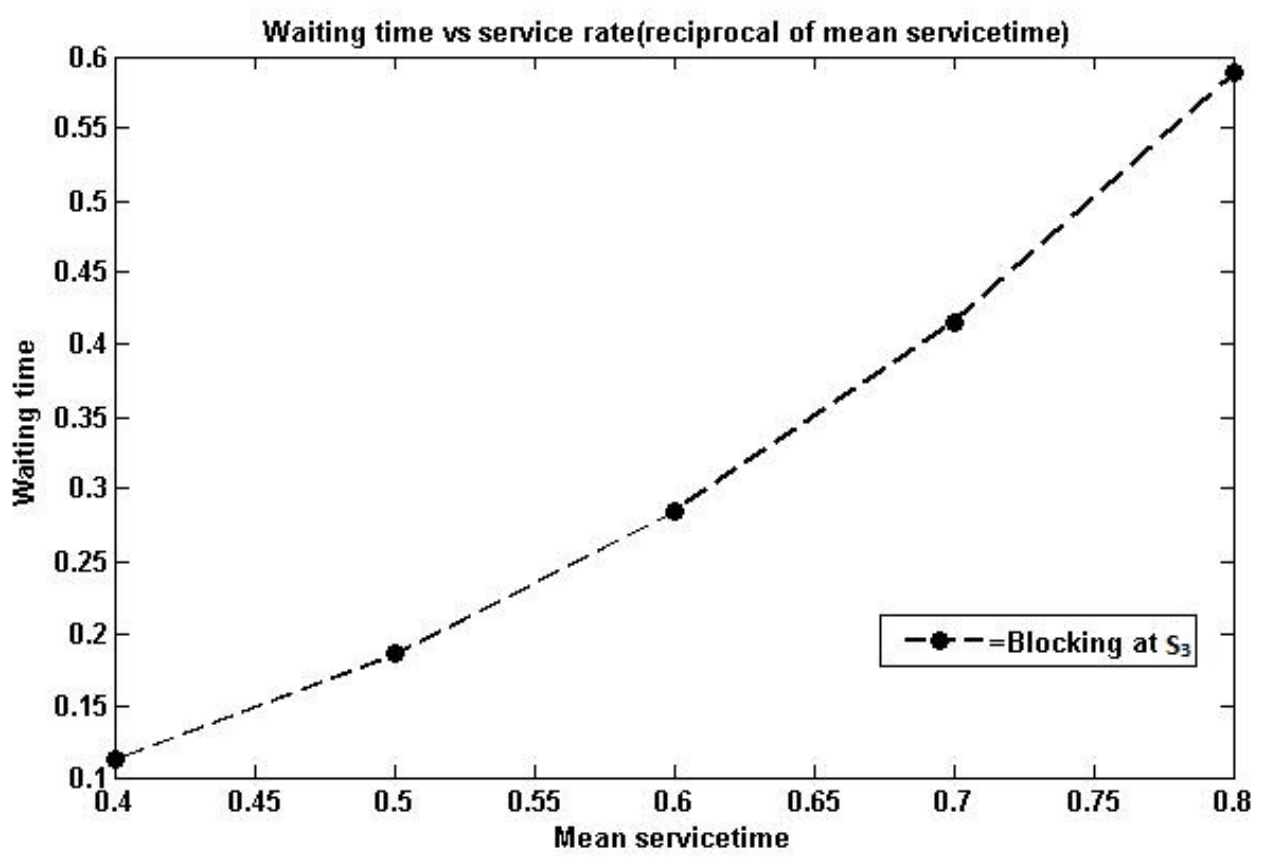

Figure 2. Congestion at station $S_{3}$.

\section{Simulation analysis}

We have used the mathematical model of the queueing network for the steady state analysis. The transient solution of this complex model is quiet tedious. However, one may seek a simulation study to know the transient behavior of the network system. The same arrival rates and service rates used for mathematical analysis are also used in the simulation study. Discrete event simulation engine "SimEvents" in Simulink Matlab library is used for the simulation study. We performed the simulation study for different simulation time viz. 100, 1000, 10000, etc. The Table 3 summarizes the typical output of the simulation study. Autocorrelation result shows that all signal values are highly correlated. The result of coefficient of variation implies that most of the signal values are efficient.

Table 3

Summary of simulation results (simulation time 1000)

\begin{tabular}{lllllll}
\hline Station & PI & Average & Minimum & Maximum & Autocorrelation & CV \\
\hline$S_{2}$ & $L_{2}^{q}$ & 0.096651 & 0.0103 & 0.1442 & 0.970253 & 0.38489 \\
& $W_{2}^{q}$ & 0.132323 & 0.0217 & 0.2028 & 0.94994 & 0.373329 \\
$S_{3}$ & $L_{3}^{q}$ & 0.876263 & 0.2214 & 1.5193 & 0.968488 & 0.261223 \\
& $W_{3}^{q}$ & 0.823248 & 0.353 & 1.2609 & 0.960683 & 0.231401 \\
$S_{4}$ & $L_{4}^{q}$ & 0.059455 & 0.0288 & 0.2325 & 0.768051 & 0.748465 \\
& $W_{4}^{q}$ & 0.10024 & 0.0692 & 0.19 & 0.608661 & 0.3751 \\
\hline
\end{tabular}

In Figure 3 we plot the waiting time to enter $S_{3}$ against the mean service time at the station. It shows a comparison between the result obtained by analytical study and simulation study. Waiting time corresponding 
to various service times of station $S_{3}$ are plotted in both the studies. It further reveals that the calculated waiting time and simulation waiting time do not differ too much. On further enhancing the simulation time we find that the results closely agree with the analytical results.

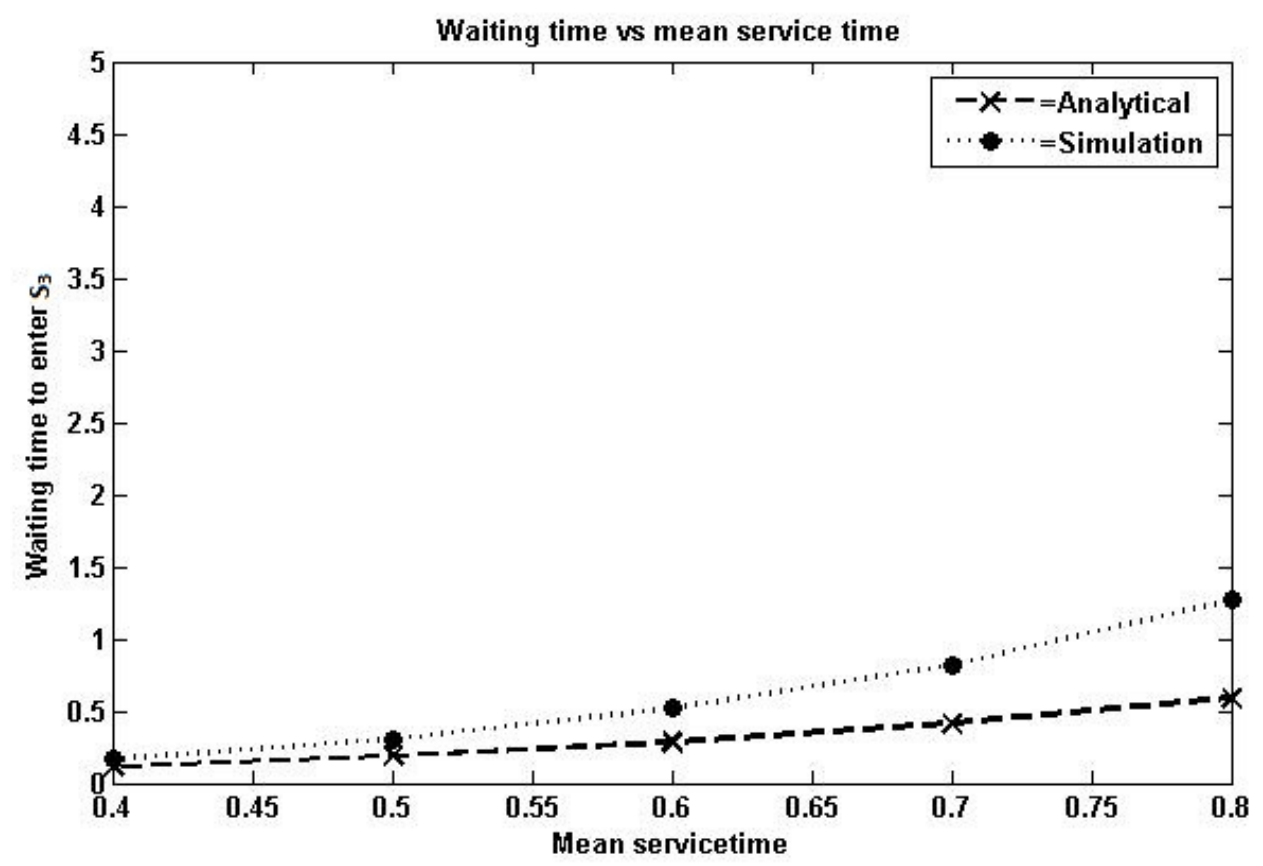

Figure 3. Simulation study versus mathematical study.

\section{Conclusion}

We studied the structural aspects of restricted open queueing network with blocking and the methodology is illustrated through a healthcare system of infertility clinic. The congestion between stations due to blocking are studied using decomposition approach. A Matlab program was developed for calculating the performance measures for an example network system. A simulation study has been conducted using SimEvents in Matlab program. As expected, there is discerning difference between the results obtained in either methods, however when the simulation time increases the results agree better. It may be noted that autocorrelation and coefficient of variation study of the simulation values show that the simulation outputs are highly correlated and more or less efficient. So from the result of simulation study we conclude that though the mathematical steady state results are easier for computation, in the presence of autocorrelated signal values, it may not be used. Although the work presented here applies the blocking approach to only a simplified model of a health care system, it can easily be extended to incorporate additional features and applied to any open restricted queueing network.

\section{References}

[1]. S.K. Bose, An introduction to queueing Systems, Kluwer academic / Plenum publishers, New York, 2002.

[2]. M.A. Cohen, J.C. Hershey and E.N. Weiss, Analysis of capacity decisions for progressive patient care hospital facilities, Health Services Research 15 (1980), 145-160.

[3]. E. El-Darzi, C. Vasilakis, T. Chaussalet and P.H. Millard, A simulation modelling approach to evaluating length of stay, occupancy, emptiness and bed blocking in a hospital geriatric department, Health Care Management Science 1 (1998), 
143-149.

[4]. G. Gross and C. Harris, Fundamentals of queueing theory, John Wiley and Sons, 1998.

[5]. J.C. Hershey, E.N.Weiss and M.A. Cohen, A stochastic service network model with application to hospital facilities, Operations Research 29 (1981), 1-22.

[6]. F.S. Hiller and R.W. Boling, Finite queues in Series with exponential or Erlang service times a numerical approach, Operations Research 15 (1967), 286-303.

[7]. G.C. Hunt, Sequential arrays of waiting lines, Operations Research 4 (1956), 674-683.

[8]. J.R. Jackson, Networks of waiting lines, Operations Research 5 (1957), 518-521.

[9]. J.R. Jackson, Jobshop-like queueing systems, Management Science 10 (1963), 131-142.

[10]. N. Koizumi, E. Kuno and T.E. Smith, Modeling patients flows using a queueing network with blocking, Health Care Manag. Sci. 8 (2005), 49-60.

[11]. R. Korporaal, A. Ridder, P. Kloprogge and R. Dekker, An analytic model for capacity planning of prisons in the Netherlands, Journal of the Operations Research Society 51 (2000), 1228-1237.

[12]. M. Manitz, Analysis of assembly/disassembly queueing networks with blocking after service and general service times, Ann. Oper. Res. 226 (2015), 417-441.

[13]. H.G. Perros and T. Altiok, Approximate analysis of open networks of queues with blocking: Tandem Configurations, IEEE Trans. Soft. Eng. 12 (1986), 450-461.

[14]. Y. Takahashi, H. Miyahara and T. Hasegawa, An approximation method for open restricted queueing networks, Operations Research 28 (1980), 594-602.

[15]. E.N. Weiss and J.O. McClain, Administrative days in acute care facilities: A queuing-analytic approach, Operations Research 35(1) (1987), 35-44.

[16]. F. Zegers-Hochschild, G.D. Adamson, J. de Mouzon, O. Ishihara, R. Mansour, K. Nygren, E. Sullivan, S. Vanderpoel, International Committee for Monitoring Assisted Reproductive Technology (ICMART) and the World Health Organization (WHO) revised glossary of ART terminology, Fertil. Steril. 92(5) (2009), 1520-1524. 